\title{
CARACTERIZACIÓN DE LOS CONOCIMIENTOS DE LAS MADRES SOBRE LA DISCAPACIDAD DE SUS HIJOS Y SU VÍNCULO CON LA DINÁMICA FAMILIAR
}

\author{
ALFA CELENE REA AMAYA*, GUADALUPE ACLE TOMASINI, AMADA AMPUDIA RUEDA, \\ MIRNA GARCÍA MÉNDEZ \\ UNIVERSIDAD NACIONAL AUTÓNOMA DE MÉXICO
}

Recibido, mayo 31/2013

Concepto evaluación, noviembre 13/2013

Aceptado, diciembre 28/2013
Referencia: Rea, A.C., Acle, G., Ampudia, A. \& García,
M. (2014). Caracterización de los conocimientos de las
madres sobre la discapacidad de sus hijos y su vínculo
con la dinámica familiar. Acta Colombiana de Psicolo-
gía, 17(1), 91-103. doi: 10.14718/ACP.2014.17.1.10

Resumen

\begin{abstract}
El propósito del estudio es establecer los conocimientos, la dinámica familiar y comunitaria de madres de hijos con discapacidad que sirvan de base para la instrumentación de programas de intervención. Participaron 42 madres con edades entre 19 y 49 años $(\mathrm{M}=31.14)$ de un Centro de Rehabilitación Infantil perteneciente a la Secretaría de la Defensa Nacional en México. Se utilizó una guía de entrevista semi-estructurada para explorar los conocimientos, la dinámica familiar y comunitaria de las familias. Las madres reportaron que no recibieron preparación de cómo tratar a sus hijos; sin embargo, ellas consideran la importancia de adquirir conocimientos especializados para entender y tratar al menor. Las madres que no tienen los conocimientos necesarios sobre la discapacidad de su hijo no son capaces de entender ni aceptar la condición del niño, conllevando a la negación del problema, inconformidad, culpa y depresión. Se observó en el estudio que una adecuada preparación de la madre después del nacimiento de su hijo, acerca de la discapacidad, puede proveer una mejor interacción y funcionamiento de estas familias. Palabras Clave: Discapacidad, Conocimientos de discapacidad, Dinámica Familiar, Dinámica comunitaria, Madres de hijos con discapacidad.
\end{abstract}

\section{CHARACTERIZATION OF THE KNOWLEDGE OF MOTHERS CONCERNING THEIR CHILDREN'S DISABILITY AND THE RELATION WITH THE FAMILY DYNAMICS}

\begin{abstract}
The purpose of this study is to enquire about the knowledge, as well as about the family and community dynamics of mothers of children with disabilities, as a basis to implement intervention programs. Participants were 42 mothers aged between 19 and 49 years $(M=31.14)$ who attended a Children's Rehabilitation Center belonging to the National Defense Secretariat in Mexico [SEDENA, for its Spanish acronym]. Semi-structured interviews were used to assess the mother's knowledge on the disability of the child and the family and community dynamics. Mothers reported receiving no training on how to treat their children, though they considered the importance of acquiring expertise to understand and treat the child. Mothers who do not have the necessary knowledge about their child's disability are not able to understand or accept the child's condition, leading to a denial of the problem, dissatisfaction, guilt and depression. It was noted in the study that proper preparation of the mother after the birth of her child, in terms of the disability can provide a better interaction and functioning of these families.

Key words: Disability, knowledge of disability, family dynamics, community dynamics, mothers of children with disabilities
\end{abstract}

\footnotetext{
* Doctora en Psicología por la Universidad Nacional Autónoma de México. Correspondencia: Oyametla Mz. 73 Lt. 13 Col. San Bernabé
} Ocotepec. C.P. 10300. Delegación. Magdalena Contreras, México, D.F. Teléfono: 01152551520 3348. celenere1982@hotmail.com 


\title{
CARACTERIZAÇÃO DOS CONHECIMENTOS DAS MÄES SOBRE A INCAPACIDADE DE SEUS FILHOS E SEU VÍNCULO COM A DINÂMICA FAMILIAR
}

Resumo

\begin{abstract}
O propósito do estudo é estabelecer os conhecimentos, a dinâmica familiar e comunitária de mães de filhos com incapacidade que sirvam de base para a instrumentação de programas de intervenção. Participaram 42 mães com idades entre 19 e 49 anos $(\mathrm{M}=31.14)$ de um Centro de Recuperação Infantil pertencentes à Secretaria de Defesa Nacional no México. Utilizou-se um roteiro de entrevista semiestruturada para explorar os conhecimentos, a dinâmica familiar e comunitária das famílias. As mães relataram que não receberam preparação de como cuidar seus filhos; porém, elas consideram a importância de adquirir conhecimentos especializados para entender e tratar ao menor. As mães que não têm os conhecimentos necessários sobre a incapacidade de seu filho não são capazes de entender nem aceitar a condição da criança, levando à negação do problema, inconformidade, culpa e depressão. Observou-se no estudo que uma preparação adequada da mãe depois do nascimento de seu filho, sobre a incapacidade, pode dar uma melhor interação e funcionamento destas famílias.

Palavras Chave: incapacidade, conhecimentos de incapacidade, dinâmica familiar, dinâmica comunitária, mães de filhos com incapacidade.
\end{abstract}

\section{INTRODUCCIÓN}

La familia funciona como una unidad interactiva e interdependiente; al ocurrir algún evento o experiencia que afecte en particular a algún miembro del grupo, perjudica a los demás miembros de la familia (Gargiulo, 2006; Núñez, 2008). Al nacer un hijo con discapacidad ocurre un impacto dentro de la familia que afecta a cada uno de los miembros que la componen; se modifican los ideales de los padres respecto a su hijo y se aumentan las fuentes de estrés tanto emocionales, como físicas, económicas y sociales (Castro et al., 2008; De Andrés \& Guinea, 2012; Maul \& Singer, 2009; Núñez, 2008; Ortega, Salguero \& Garrido, 2007; Pérez \& Lorenzo, 2007; Torres \& Maia, 2009; Vargas et al., 2012).

El futuro del niño con discapacidad depende en gran medida de la forma en que la familia percibe y afronta la discapacidad. La respuesta de los miembros de ésta dependerá de diversos factores tales como los rasgos de personalidad, el estado de ánimo de cada uno de ellos, el nivel cultural y de estudios o la gravedad del diagnóstico y pronóstico (Madrigal, 2007). Es posible también que afecte cómo los padres evalúan su vida familiar, lo cual puede influir en la percepción de todos los miembros de la familia (Gargiulo, 2006).

Las instituciones de salud son de suma importancia en el acompañamiento y en la elaboración de un adecuado diagnóstico para las familias de niños con discapacidad. El peregrinaje en busca de un diagnóstico fiable es una etapa difícil de superar, no sólo porque en ocasiones puede durar mucho tiempo sino por el desgaste físico, psicológico y emocional que genera en los padres; es importante que éstos tengan el diagnóstico de su hijo lo antes posible, ya que conocerlo puede ser el principio de la aceptación de su realidad y de permitirse comenzar a actuar (Cagran, Schmidt \& Brown, 2011; Martínez \& Bilbao, 2008). La poca claridad frente al diagnóstico, así como el acompañamiento que se hace a los padres, resulta crucial para asumir la discapacidad en forma integral; al tener un diagnóstico fiable las familias logran albergar deseos positivos en relación con la discapacidad. Así mismo, estas familias se unen ante la adversidad, aparece en ellas la cohesión y debido a la falta de soporte psicosocial, la actitud de resignación es evidente. La situación de la persona con discapacidad se vuelve más difícil a medida que la familia comienza a creer que ésta no puede alcanzar mejores niveles de vida a causa de sus limitaciones cognitivas y sociales. Por ello surgen otros trastornos psicopatológicos en dicha persona, lo cual termina por afectar nuevamente a la familia al no poseer los recursos ni los conocimientos necesarios acerca del problema (Mora, Córdova, Bedoya \& Verdugo, 2007). $\mathrm{Si}$ a la familia se le brinda apoyo profesional y los conocimientos relacionados con la discapacidad para que acepte la condición del integrante que la presenta, se logrará además que dicha familia identifique las habilidades de esta persona, mejorando así su calidad de vida y la dinámica familiar.

Es importante brindar a los padres la preparación respecto al conocimiento y aceptación de la discapacidad de su hijo, debido a que la mayoría de las veces ellos consideran que no poseen los elementos necesarios para lidiar con la experiencia de tener un hijo con discapacidad. Cuando las familias adquieren los conocimientos y comprenden lo que representa tener un miembro con esta condición es posible observar cómo asumen el diagnóstico y logran establecer una relación con el entorno (Rodríguez, Alvarado \& Moreno, 2007). Los conocimientos permiten a los padres una mayor aceptación y comprensión sobre el diagnóstico de su hijo (Huang, Tsai \& Kellet, 2011; Peralta \& Arellano, 2010). 
Un factor que dificulta o facilita la búsqueda de recursos para el niño con discapacidad es el tipo de interpretación que realizan los padres al conocer dicho diagnóstico. Torres \& Maia (2009) entrevistaron a madres brasileñas de hijos con síndrome de Down y evidenciaron que la falta de información concreta y científica respecto al diagnóstico del síndrome afecta significativamente la manera en que las madres interpretan la problemática del niño. Por el contrario, tener acceso a dicha información podría ayudarles a comprender cómo lidiar con la discapacidad de su hijo. El diagnóstico puede tornarse más positivo dependiendo de la forma en que sea transmitido a la familia y de cómo se maneje la atención ofrecida al niño. Acle, Roque, Zacatelco, Lozada \& Martínez (2007) describen la importancia de realizar diagnósticos diferenciales precisos para dar la atención adecuada a los menores con discapacidad y brindar así un apoyo apropiado a sus padres.

La aceptación e integración de las personas con discapacidad a la sociedad es un aspecto importante. Por ello, es primordial analizar qué pasa con la familia, cómo impacta el hecho de tener un integrante con alguna discapacidad y qué factores intervienen dentro del núcleo familiar, para ver cómo se da la relación con esa persona (Covarrubias, 2008). El intercambio continuo de información entre padres y profesionales reduce el sentimiento de culpa que sienten los padres y puede contribuir positivamente a mejorar la calidad de vida de las familias con hijos que presenten alguna discapacidad (Masood, Turner \& Baxter, 2007).

A nivel mundial existen 500 millones de personas con alguna discapacidad, lo que equivaldría al $10 \%$ de la población; de este porcentaje, 140 millones son niños, otros 300 millones viven en países en desarrollo y únicamente el $2 \%$ de la población tiene acceso a asistencia médica y servicios adecuados (Rolander, Montes \& Culebro, 2008; United Nations Statistics Division, 2009). La situación en México respecto a la discapacidad está a cargo de programas como El Programa de Atención a Personas con Discapacidad e instituciones como el Sistema Nacional Para El Desarrollo Integral de la Familia (DIF) Nacional, el cual presta servicios de rehabilitación integral a la población con discapacidad o en riesgo de presentarla, contribuyendo a su integración social. Las familias mexicanas de hijos con discapacidad no cuentan con la atención suficiente para las necesidades derivadas de la discapacidad; se requiere de apoyos que les permitan acceder a los servicios de salud, rehabilitación y educación. La falta de atención está determinada por diversas causas, entre las que se incluyen la carencia de servicios, pero también la ausencia de información de los padres y la incorporación a un sistema de canalización oportuno (Gobierno del Distrito Federal,
DIF-DF, UNICEF, 2006). En México se desconoce el número de familias que cuentan, al menos, con una persona en condición de discapacidad (Programa Nacional para el Desarrollo de las Personas con Discapacidad 2009-2012, 2009) por lo cual es importante considerar si las familias mexicanas que tienen hijos con esta característica se encuentran inscritas en algún programa, para darles el apoyo $\mathrm{y}$ atención necesarios.

El total de personas con discapacidad en el país es de 5.739.270, de acuerdo con el último censo realizado, lo que equivale al $5.1 \%$ de la población; de esta población 520.369 son niños con edades entre 0 y 14 años, equivalente al $1.6 \%$ de la población total con discapacidad (Instituto Nacional de Estadística y Geografía - INEGI, 2010). Por ello es primordial continuar con investigaciones e intervenciones que les proporcionen a las familias de niños con discapacidad un apoyo integral, y que a su vez les permita tener mayor conocimiento sobre esta problemática, así como establecer una adecuada dinámica familiar y comunitaria, resolver las dudas y temores respecto a su hijo con discapacidad, y lograr así una mejor calidad de vida para todos los miembros que integran la familia. El objetivo de este estudio es el de explorar y caracterizar los conocimientos, la dinámica familiar y comunitaria de madres de hijos con discapacidad, cuyo resultado permita instrumentar programas de intervención más apropiados a las necesidades de estas madres.

\section{MÉTODO}

\section{Participantes}

La elección de las participantes se llevó a cabo mediante un muestro probabilístico, ya que se eligió una muestra de madres cuyos hijos fueron diagnosticados en el Centro de Rehabilitación Infantil con discapacidades del lenguaje, autismo, físicas y de la salud. (Hernández, Fernández \& Baptista, 2010). Se tomó como criterio de inclusión que la familia estuviera constituida por padre y madre. Como criterios de exclusión no se tuvieron en cuenta las madres que no desempeñaban el rol de cuidadoras primarias y que no contestaran el $80 \%$ de la entrevista.

La muestra se conformó con 42 madres de niños con algún diagnóstico de discapacidad que solicitaron atención médica y psicológica para su hijo en el servicio externo del Centro de Rehabilitación Infantil (CRI) perteneciente a la Secretaría de la Defensa Nacional (SEDENA) con edades entre 19 y 49 años, $(M=31.14 ; \mathrm{DE}=7.78)$. En relación al grado militar, el $95.2 \%$ de las madres no poseía ningún grado militar y únicamente el $4.8 \%$ reportó ser sargento primero o cabo. El nivel de escolarización de la muestra se presenta en la tabla 1. 
$\underline{\text { Tabla } 1}$

Nivel de escolarización de las madres

\begin{tabular}{lc}
\hline \multicolumn{1}{c}{ Nivel de escolarización } & Porcentaje \\
\hline No tiene estudios & 4.8 \\
Primaria & 9.5 \\
Secundaria & 45.2 \\
Bachillerato & 23.8 \\
Carrera Técnica & 2.4 \\
Licenciatura & 14.3 \\
\hline Total & 100 \\
\hline
\end{tabular}

Es importante indicar que la mayoría se dedica al hogar (81\%), el 9.6\% realiza actividades tales como distribuir suplementos alimenticios, ser dependiente de una juguería o empleada de maquila de cartón; el $4.8 \%$ ejerce como profesoras de educación primaria o secundaria; y el 2.4\% trabaja como sastre u oficinista en SEDENA.

Respecto al sexo de los hijos con discapacidad, el 52.4\% era masculino y el $47.6 \%$ femenino. La edad de los menores osciló entre uno y trece años $(\mathrm{M}=4.55 ; \mathrm{DE}=3.086)$. El tipo de discapacidad se muestra en la tabla 2.

Tabla 2

Tipo de discapacidad de los menores según diagnóstico de SEDENA

\begin{tabular}{lc}
\hline \multicolumn{1}{c}{ Discapacidad } & Porcentaje \\
\hline Acondroplasia & 2.4 \\
Autismo & 4.8 \\
Disgenesia en el cuerpo calloso y amplitud del espacio & 2.4 \\
subaracnoideo bifrontal & \\
Dislalia & 2.4 \\
Distrofia muscular de Duchenne & 2.4 \\
Epilepsia & 2.4 \\
Hemiparesia & 4.8 \\
Hipoacusia & 2.4 \\
Luxación de la cadera, congénita & 2.4 \\
Parálisis cerebral infantil (PCI) & 16.7 \\
Pie equinovaro bilateral & 2.4 \\
Problemas de coordinación motora & 2.4 \\
Retraso global en el neurodesarrollo & 7.2 \\
Retraso mental moderado & 2.4 \\
Secuela de encefalopatía hipóxica, neumopatía crónica & 2.4 \\
del lactante, hispoxicoisquemica y gastrotomia & \\
Síndrome de West & 2.4 \\
Tortícolis congénita, Síndrome de Chiari y de Klepperflei & 2.4 \\
Trastorno por déficit de atención e hiperactividad (TDAH) & 9.5 \\
Trastorno del lenguaje & 19 \\
Trastorno generalizado del desarrollo & 7.1 \\
\hline Total & 100 \\
\hline
\end{tabular}

\section{Escenario}

Las aplicación de la guía de entrevista semiestructurada para padres se realiza en un Centro de Rehabilitación Infantil (CRI) perteneciente a la Secretaría de la Defensa Nacional (SEDENA). Los servicios que se prestan son gratuitos y exclusivamente para el personal que labora en SEDENA.

\section{Tipo de estudio}

El tipo de estudio es transversal y de campo, pues se recolectaron los datos en un solo momento con el propósito de observar y describir los fenómenos tal como se dan en su contexto natural para posteriormente analizarlos. (Hernández et al., 2010).

\section{Diseño}

El tipo de diseño es exploratorio y descriptivo, pues permite obtener información respecto a un contexto en particular y especificar características de dicho fenómeno en una sola muestra extraída de una población determinada, que en este caso es de madres con un hijo con discapacidad (Hernández et al., 2010).

\section{Instrumento}

Guía de entrevista semiestructurada para padres de hijos con discapacidad (Rea, Ampudia y Acle; 2010) constituida por 139 reactivos organizados en cuatro áreas: a) Ficha de identificación y datos sociodemográficos (29); b) Conocimiento de la discapacidad (76); c) Dinámica familiar (22); y d) Dinámica comunitaria (12). Esta guía se validó mediante un proceso de interjueces con profesionales vinculados a la educación especial y médicos de SEDENA, cuyo acuerdo fue de $95 \%$.

\section{Procedimiento}

Se realizaron los trámites necesarios en la Secretaría de la Defensa Nacional (SEDENA) para obtener la autorización oficial y acudir a realizar las entrevistas en las instalaciones del Centro de Rehabilitación Infantil (CRI), al cual se asistió durante 4 meses, de lunes a viernes de las 8:00 a las 14:00 horas. De las madres que asistieron con sus hijos a consulta en el área de psicología y de consulta externa del CRI se seleccionaron aquellas que cubrían los criterios de inclusión y aceptaban participar voluntariamente en el estudio.

Al inicio de la entrevista se le explicó a la madre el propósito de ésta y en caso de tener alguna duda se le aclaraba en el momento; la transcripción de las respuestas de las madres se hizo simultáneamente a la entrevista; la duración de cada entrevista fue aproximadamente de una hora con 30 minutos. La información obtenida en la entrevista se anexó al expediente del menor y se guardó una copia en 
los archivos del área de psicología para que el personal de esta área pudiera utilizarlos en posteriores citas del menor.

Análisis de datos

De las áreas que componen la guía se agruparon las respuestas de acuerdo a las siguientes categorías:

1. Conocimiento de la discapacidad. Interesa saber cuándo, cómo y quién le informó de la discapacidad de sus hijos. A nivel conceptual, el conocimiento de la discapacidad es el conjunto de información obtenida mediante el aprendizaje o experiencia respecto a la discapacidad de sus hijos. En este instrumento se define como la información que las madres poseen respecto a los antecedentes pre y perinatales del menor, así como las emociones referentes a la discapacidad.

2. Dimensión dinámica familiar. Interesa saber cómo es el apoyo de la pareja y qué tipo de apoyo y de quiénes se ha recibido éste por parte de la familia extensa. La dinámica familiar, a nivel conceptual, se define como los aspectos interaccionales que surgen en el interior de la familia, donde cada miembro está ligado a los demás por un lazo de parentesco y/o afectividad, comunicación, autoridad, normatividad y por la distribución de roles sociales de los miembros (Sarmiento, 1994); en la guía de entrevista semiestructurada se define como la información respecto a la interacción y apoyo entre la pareja para el cuidado del menor, así como el tipo de apoyo y conocimiento de la discapacidad que proporciona la familia extensa.

3. Dimensión dinámica comunitaria. Importa saber si la madre posee redes de apoyo con padres de hijos con discapacidad y la relación del niño con la comunidad. A nivel conceptual, la dinámica comunitaria es un conjunto de acciones establecidas e iniciativas de los miembros de una comunidad, individualmente y en colectivos (Ulloa, 2010); en este instrumento se define como la relación que la madre percibe con la comunidad y las redes de apoyo que la madre posee con padres de hijos con discapacidad.

\section{RESULTADOS}

Conocimiento de la discapacidad. Respecto a los antecedentes prenatales, es importante conocer si las madres identifican alguna etiología de la discapacidad de sus hijos, por lo que se exploró si durante el embarazo hubo algún riesgo que pudiera ser el motivo de la discapacidad del menor. A continuación se presentan los resultados: Sólo en diez de los casos (24\%) se presentaron complicaciones, las cuales conjuntamente con el tipo de discapacidad del menor se muestran en la tabla 3.

Tabla 3

Tipo de complicaciones y diagnóstico de discapacidad realizado por los neuropediatras del CRI

\begin{tabular}{|c|c|c|}
\hline Tipo de complicación & Porcentaje & Diagnóstico de discapacidad \\
\hline $\begin{array}{l}6^{\circ} \text { mes dolor abdominal, reposo por una semana. Último trimestre el } \\
\text { bebé dejó de crecer. }\end{array}$ & 2.4 & $\begin{array}{l}\text { Trastorno por déficit de atención e hiperactividad } \\
\text { (TDAH) }\end{array}$ \\
\hline $8^{\circ}$ mes circular de cordón umbilical & 2.4 & Acondroplasia \\
\hline $\begin{array}{l}\text { Al principio sangrado y no podía hacer ningún esfuerzo. Se estaba } \\
\text { pasando el embarazo por una o dos semanas }\end{array}$ & 2.4 & Retraso global en el neurodesarrollo \\
\hline $\begin{array}{l}\text { Amenaza de parto prematuro a las } 29 \text { semanas y circular de cordón } \\
\text { umbilical }\end{array}$ & 2.4 & Trastorno generalizado del desarrollo \\
\hline Amenazas de aborto y crecimiento de la placenta insuficiente & 2.4 & Retraso global en el neurodesarrollo \\
\hline Caída de un puente peatonal & 2.4 & Hipoacusia \\
\hline $\begin{array}{l}\text { Embarazo gemelar. Internan a las } 26 \text { semanas para poder llegar a } \\
\text { las } 30 \text { semanas e interrumpir el embarazo ya que uno de los bebés } \\
\text { presentaba poligramas. }\end{array}$ & 2.4 & Parálisis cerebral infantil (PCI) \\
\hline $\begin{array}{l}\text { Incontinencia urinaria; contracciones y expulsión de líquido } \\
\text { amniótico }\end{array}$ & 2.4 & Parálisis cerebral infantil (PCI) \\
\hline $\begin{array}{l}\text { Primeras } 9 \text { semanas hemorragias y en la semana } 32 \text { desprendimiento } \\
\text { de placenta }\end{array}$ & 2.4 & $\begin{array}{l}\text { Secuela de encefalopatía hipóxica, neumopatía crónica, } \\
\text { hispoxicoisquemica. }\end{array}$ \\
\hline $\begin{array}{l}\text { Sangrado por esfuerzo al } 2^{\circ} \text { mes y al } 6^{\circ} \text { mes amenaza de aborto por } \\
\text { lo que permanece el reposo el resto del embarazo }\end{array}$ & 2.4 & $\begin{array}{l}\text { Disgenesia en el cuerpo calloso y amplitud del espacio } \\
\text { subaracnoideo bifrontal }\end{array}$ \\
\hline Total & 24 & \\
\hline
\end{tabular}


Tabla 4

Problema durante el parto y diagnóstico de discapacidad

\begin{tabular}{lcl}
\hline \multicolumn{1}{c}{ Tipo de complicación } & Porcentaje & \multicolumn{1}{c}{ Diagnóstico de discapacidad } \\
\hline Caída de un puente peatonal & 2.4 & Hipoacusia \\
Desprendimiento de placenta & 2.4 & Secuela de encefalopatía hipóxica, neumopatía crónica, hispoxicoisquemica. \\
No existían problemas, únicamente se sintió mal & 2.4 & Parálisis cerebral infantil (PCI) \\
Nunca le dijeron & 2.4 & Problemas de coordinación motora \\
Riesgos que presentaban los bebés & 2.4 & Parálisis cerebral infantil (PCI) \\
Rompimiento de fuente & 9.5 & Parálisis cerebral infantil (PCI) (2); Disgenesia en el cuerpo calloso y \\
& & amplitud del espacio subaracnoideo bifrontal; y Distrofia muscular de \\
No sabe & 2.4 & Puchenne \\
\hline Total & 23.9 & \\
\hline
\end{tabular}

La información obtenida coincide con lo establecido por la literatura, la cual señala cuáles son los factores que pueden ser causa de discapacidad; no obstante, el tipo de complicaciones prenatales de esta muestra en particular se relaciona con factores relevantes de riesgo (Zipper \& Simeonsson, 2004), tales como la etiología de la discapacidad y la forma en que son informados los padres (Gargiulo, 2006). En uno de los casos en que se le informa a la madre la discapacidad antes del nacimiento se aprecia primero el rechazo de la situación de la hija y la aceptación posterior, lo que se ilustra en el siguiente comentario:

"Al principio la rechazaba y no quería que naciera para que no sufriera, basándome en la sociedad, en cómo la iban a tratar y cómo iba a ser. Me explicaban que era lo mejor. Pero la niña tenía muchas ganas de nacer, le echó muchas ganas y el que ahora ría nos motiva para sacarla adelante" (Mamá de niña con acondroplasia).

Se consideran también los antecedentes perinatales porque una complicación en el momento del nacimiento puede ser causa de la discapacidad (Gargiulo, 2006; Zipper \& Simeonsson, 2004); en este sentido se encontró que en el $76.2 \%$ de los casos el parto fue a término, pero en el $23.9 \%$ de éstos se presentó algún tipo de problema; las causas se muestran en la tabla 4.

Las respuestas que proporcionan las madres en relación con el diagnóstico de los menores no concuerdan con la etiología de las discapacidades de los mismos; podemos observar la falta de información que ellas poseen desde el nacimiento del menor. La literatura señala la importancia de que se brinde información de la discapacidad a los padres en la fase prenatal o dentro de las primeras 24 a 48 horas después del parto para que ellos estén preparados y disminuya así la tensión, angustia, estrés, shock, entre otras emociones que las madres presentan (Torres \& Maia, 2009)

La información obtenida con respecto al diagnóstico de la discapacidad tiene el objetivo de conocer en qué momento se les informa a las madres que su hijo presenta una discapacidad, quién les informa, cómo y cuál es la reacción de ellas ante dicho diagnóstico. Se analizan los resultados a partir de cuatro aspectos: a) en qué momento se entera la madre de la discapacidad del menor, b) quién le informa; c) cómo le informan de la discapacidad; y d) cuáles son las reacciones de la madre. A continuación se presentan los datos de cada aspecto.

Tabla 5

Edad del menor al momento del diagnóstico

\begin{tabular}{lc}
\hline \multicolumn{1}{c}{ Discapacidad } & Porcentaje \\
\hline Al nacer & 21.4 \\
Antes de los 10 meses & 21.4 \\
Entre el año o año y medio & 14.3 \\
Entre los 2 y 3 años & 19 \\
Entre los 4 y 6 años & 16.7 \\
Entre los 7 y 8 años & 4.8 \\
A los 13 años & 2.4 \\
\hline Total & 100 \\
\hline
\end{tabular}

En la tabla 5 se puede observar una variabilidad en la edad en que se hace el diagnóstico y que tiene que ver con el tipo de discapacidad; casi en la mitad de los casos se les informa de ésta dentro de los primeros meses de nacimiento, lo cual se relacionan con la presencia de discapacidades severas que se diagnostican y observan inmediatamente; la otra mitad se refiere a aquellos tipos de discapacidades 
que se van observando cuando el menor no cumple con las metas de desarrollo a una determinada edad (Masten \& Powell, 2007). Se ilustra en las siguientes respuestas de las madres:

"Me mandan a llamar de la escuela y me dicen que tenía un problema de lenguaje y que necesitaba tratamiento" (Mamá de niña con trastorno de lenguaje).

"El doctor que la revisaba desde bebé nos dice que su comportamiento no es normal y que probablemente tenga autismo y nos canaliza al CRI para hacerle más estudios" (Mamá de niña con autismo).

"Lo llevamos al doctor porque cuando entró al kínder nos decían que había que cortarle la lengua para poder hablar y ya en el CRI nos dijeron que el problema era un retraso en el lenguaje" (Mamá de niño con trastorno de lenguaje).

Lo anterior se encuentra también vinculado a quién y cómo se proporciona el diagnóstico. En la tabla 6 se observa que principalmente son los profesionales quienes informan a las madres de la discapacidad del menor; sin embargo, la información no es tan precisa como se esperaría y en algunos casos únicamente se les informa el diagnóstico del menor.

Tabla 6

Quién le informa el diagnóstico de discapacidad

\begin{tabular}{lc}
\hline \multicolumn{1}{c}{ Quién informa } & Porcentaje \\
\hline Centro de Rehabilitación Infantil & 4.8 \\
Especialista (doctor, pediatra, neurólogo o & 76.2 \\
neonatólogo) & 4.8 \\
La maestra de la escuela & 7.1 \\
Lo observan ellas & 2.4 \\
Psicólogo del hospital & 2.4 \\
Trabajadora social de la escuela & 2.4 \\
Una prima de la madre & 100 \\
\hline Total &
\end{tabular}

Stoner y Angell (2006) señalan la importancia que tiene la relación dinámica de los profesionales con los padres para que se construya la confianza entre ambos, pues los padres desean y buscan un fuerte lazo de confianza con los profesionales ya que consideran que la relación podría beneficiar a sus hijos; sin embargo, esta relación dinámica no parece existir en el CRI, lo cual se puede observar en lo que reportan las madres entrevistadas. Algunas de las respuestas ilustran esta situación:
"Me dicen que estaba mal y que se iba a quedar en el hospital" (Mamá de niño con hipoacusia).

"Nosotros comenzamos a ver el diagnóstico en las recetas, le preguntamos al doctor y el nos confirma que tenía el síndrome de West" (Mamá de niño con síndrome de West).

El cómo se les informa tampoco contribuye al establecimiento de esa relación de confianza (véase tabla 7)

Tabla 7

Cómo le informa el diagnóstico de discapacidad

\begin{tabular}{lc}
\hline \multicolumn{1}{c}{ Cómo informan } & Porcentaje \\
\hline Explicación breve & 14.4 \\
$\begin{array}{l}\text { Realizan más estudios para confirmar el } \\
\text { diagnóstico sin que aun le informen }\end{array}$ & 16.8 \\
Únicamente informan el diagnóstico & 57.6 \\
\hline Total & 100 \\
\hline
\end{tabular}

Se puede observar que en más de la mitad de los casos se da sólo el diagnóstico de la discapacidad, dejan muchas dudas a las madres y posiblemente por la carencia de información ellas se crean falsas expectativas respecto a su hijo con discapacidad; el $11.2 \%$ no contesta. Coincidiendo con el estudio realizado por Anderson, Elliott \& Zurynski (2013), la literatura señala que si se les informa a los padres acerca de la discapacidad con métodos más apropiados, éstos podrían disminuir la sensación de impotencia que experimentan frente al diagnóstico (Marcheti, Noda \& Ferreira, 2008). Las respuestas de las madres sobre cuál es la forma en que les informan el diagnóstico de discapacidad se presentan a continuación:

"Me dicen en un centro de especialistas de la columna, que su cerebro ya no se puede desarrollar y que necesitaba mucha terapia. En el CRI me habían dicho que estaba normal la primera vez" (Mamá de niño con parálisis cerebral infantil).

"Me mandan con el cardiólogo y el ortopedista para hacerle más estudios y de ahí me dieron el diagnóstico. Lo traía al pediatra pero me decía que estaba bien" (Mamá de niño con problemas de coordinación motora).

"Que eran neuronas que estaban dormidas y que poco a poco iban a despertar" (Mamá de niño con retraso generalizado del desarrollo). 
"Sorprendida porque no sabía. No me dieron mucha información, solo dijeron que es hiperactivo, llévelo aquí" (Mamá de niño con trastorno por déficit de atención e hiperactividad).

Ante este tipo de diagnósticos e información la manera en que las madres reaccionaron se presenta en la tabla 8 .

Tabla 8

Reacción de la madre ante el diagnóstico de discapacidad

\begin{tabular}{lc}
\hline \multicolumn{1}{c}{ Reacción } & Porcentaje \\
\hline Aceptación y dolor & 12 \\
Confusión y mal & 14 \\
Desconfianza y esperanza & 9.4 \\
Despreocupación & 4.8 \\
Preocupación y culpa & 2.4 \\
Tristeza & 57.4 \\
\hline Total & 100 \\
\hline
\end{tabular}

La aceptación y dolor que presentaron las madres ante la discapacidad del menor se pueden ejemplificar con las siguientes respuestas:

"Cuando nos dicen leucemia, pensé en muerte, al saber que había solución sólo pensaba en sacar adelante lo que nos tocó" (Mamá de niña con parálisis cerebral infantil).

"Me puse a pensar que sí estaba triste, pero después contenta porque nació y está conmigo" (Mamá de niño con pie equino varo).

Las reacciones negativas de las madres ante la discapacidad de sus hijos como la confusión, la tristeza, la culpa, entre otras, se ilustran con las siguientes respuestas:

"Sentí que el mundo se me caía encima, uno nunca piensa que su hijo va a nacer mal, me costó mucho que ella hubiera nacido con ciertos problemas" (Mamá de niña con tortícolis congénita, síndrome de Chiari y de Klepperflei).

"Todo se derrumbó, se nos vino abajo el proyecto de vida y de mi parte tener más el apoyo hacia él. Me sentí más mal yo que mi esposo" (Mamá de niño con distrofia muscular de Duchenne).

"Triste, pensé muchas cosas. Me preguntaba a que se debía todo esto" (Mamá de niño con retraso generalizado del desarrollo).
"Me quede en shock, yo lo veía normal, nunca imagine que tuviera ese problema" (Mamá de niño con retraso mental).

"Mal, porque decía que no era posible. Me metí a internet y llegamos a decir que la doctora está loca" (Mamá de niño con autismo).

Una vez que las madres son informadas de la discapacidad de sus hijos, es importante conocer si tienen o no conocimiento sobre ella y si lo tienen cuál es ese conocimiento. Cabe señalar que sólo el 50\% de las madres respondió tener información sobre la discapacidad de su hijo. No obstante, de la muestra total es importante señalar que el $83.3 \%$ no recibió ningún tipo de información ni de preparación después de conocer el diagnóstico del menor; el $16.7 \%$ buscó, por su cuenta, obtener información que les ayudara, ya fuera asistiendo a la escuela para padres que dentro del CRI brindaba un pedagogo a todas las madres, independientemente de la discapacidad; buscando ellas mismas información, o comprando material para que sus hijos identificaran familiares o animales. De hecho, el $100 \%$ de las madres considera necesario tener conocimiento especializado acerca de la discapacidad de sus hijos. Estudios como los realizados por Cagran et al. (2011); Marcheti et al. (2008); Martínez \& Bilbao (2008); Torres \& Maia, (2009) señalan la importancia de que las madres reciban la información en el menor tiempo posible y de manera completa para que logren superar, aceptar y observar la discapacidad del menor de forma más positiva, lo que en esta muestra no se encontró.

De lo anteriormente descrito se desprenden las emociones y preocupaciones de las madres respecto al futuro de sus hijos, a los sentimientos que se producen en ellas en el momento en que les informan de la discapacidad de sus hijos; se observa que la mayoría se pone mal, se sienten frustradas, preocupadas y tristes (véase Tabla 9).

Tabla 9

Sentimientos ante la discapacidad del menor

\begin{tabular}{lcc}
\hline & Sentimientos & Porcentaje \\
\hline Bendecida & 2.4 \\
Culposa & 4.8 \\
Desconcertada & 4.8 \\
Desdichada & 2.4 \\
Frustrada & 14 \\
Mal & 43.2 \\
Preocupada & 7.2 \\
Tranquila & 14 \\
Triste & 7.2 \\
\hline Total & 100 \\
\hline
\end{tabular}


Las respuestas negativas de las madres, como el sentirse mal, frustradas, desesperanzadas o preocupadas ante la condición de discapacidad de sus hijos se muestran a continuación:

"No quiero llamarlo tristeza, más bien es preocupación por la situación. Yo veo a mi hija normal, nunca la vi diferente, a últimas fechas dan las características del autismo y me percato que coinciden con las de mi hija, creía que su comportamiento era porque estaba mimada y los gestos o movimientos eran herencia de papá" (Mamá de niña con autismo).

"Me sentí mal y me sigo sintiendo mal" (Mamá de niña con tortícolis congénita, síndrome de Chiari y de Klepperflei).

"Básicamente un rechazo cuando él no sabe adecuadamente las reglas, es bastante complicado; yo lo entiendo pero los demás no y esto me hace sentir mal" (Mamá de niño con trastorno generalizado del desarrollo).

"Fue una caída muy cruel, porque como todo padre, queríamos una hija sin problemas pero hay que tratar de sacarla adelante, no queda otra..." (Mamá de hija con parálisis cerebral).

"Mal, porque no podía ayudarlo, porque no podía hacer nada" (Mamá de niño con trastorno de lenguaje).

"Pues mal, en cuestión de que esperaba que saliera todo bien y de repente está enferma y pues yo creía que ya había acabado con mi otro hijo, ¿ahora que iba a pasar? Solo decepción, de que ya nos tocó otro igual pues ya ni modo" (Mamá de niña con trastorno generalizado del desarrollo).

Las respuestas de las madres concuerdan con estudios realizados por Berástegui \& Gómez-Bengoechea (2006); Burrows, Adams \& Spiers (2008); Castro et al. (2008); Ching (2009); Maul \& Singer (2009); Núñez (2008); Rosenzweig, Brennan, Huffstutter \& Bradley (2008); Soto (2008); Soulé (2009); Strnadová (2006); Vargas et al. (2012) quienes señalan el impacto que tiene en la familia el nacimiento del menor con discapacidad.

En un porcentaje menor las madres presentaron emociones positivas o se sentían bendecidas ante la discapacidad, algunas de sus respuestas se presentan a continuación:

"Normal, como cualquier otra persona" (Mamá de niño con parálisis cerebral infantil).
"Ya después de toda la revolución llega la calma y te preparas para lo que viene, para todo. Tratamos de vivir al día con él" (Mamá de niño con secuela de encefalopatía hipóxica, neumopatía crónica del lactante, hispoxicoisquemica y gastrotomía).

"Bendecida" (Mamá de niña con parálisis cerebral infantil).

Los sentimientos expresados en el momento de la entrevistas se presentan en la tabla 10. Cabe señalar que después del tiempo no se observan modificaciones con respecto a los sentimientos presentes en el momento en que se les dice que sus hijos tienen discapacidad; por el contrario, pasado el tiempo las preocupaciones se incrementan.

$\underline{\text { Tabla } 10}$

Actualmente que emociones le provoca la discapacidad

\begin{tabular}{lcc}
\hline & Sentimientos & Porcentaje \\
\hline Contenta & 12 \\
Desesperada & 9.6 \\
Despreocupada & 21.6 \\
Esperanzada & 9.6 \\
Frustrada & 14 \\
Preocupada & 7 \\
Tranquila & 7 \\
Triste & 19.2 \\
Total & 100 \\
\hline
\end{tabular}

Las expresiones de las madres ante estas emociones negativas actuales se presentan a continuación:

"Me preocupa que yo pueda estar siempre con él, que le pueda durar toda la vida. Que no me preocupe que pueda pasar con él cuando yo no esté" (Mamá de niño con trastorno generalizado del desarrollo).

"Desesperación, porque no puedo comunicarme con él. Quiero ayudarlo, tratar de que me diga qué quiere, pero sí, es muy difícil, pues no me entiende" (Mamá de niño con hipoacusia).

"Depresión, porque a veces estoy ya ni sé cómo. Si le digo, si no le digo, de todas formas no hace caso..." (Mamá de niña con trastorno de lenguaje).

"Siento ataques de ansiedad, falta de respiración, taquicardia, dolores de cabeza, histeria. Necesito ir al 
psiquiatra" (Mamá de niño con secuela de encefalopatía hipóxica, neumopatía crónica del lactante, hispoxicoisquémica y gastrotomia).

"Me sigo sintiendo frustrada, atada de manos; porque como mujer y como persona no me siento realizada. No es que reniegue de mi hija pero así me siento...” (Mamá de niña con parálisis cerebral infantil).

Algunas verbalizaciones de las emociones positivas son:

"De primero me deprimí mucho, no sabía cómo reaccionar pero después me di cuenta que la niña necesitaba mucho apoyo y pues decidí darle todo mi apoyo para que saliera adelante" (Mamá de niña con trastorno generalizado del desarrollo).

"Ya lo asimilamos y ya es tratar de ser constante. Y ya vimos muchas ventajas de que no falte a las terapias y seguirla estimulando porque no pierdo la fe de que algún día camine..." (Mamá de niña con parálisis cerebral infantil).

"Más tranquila porque ya no es tanto que puedo hacer para ayudarla sino ahora es que tengo que hacer para ayudarla" (Mamá de niña con trastorno de lenguaje).

De lo anterior se deriva la percepción del futuro de sus hijos, el 57\% lo ve de manera realista, como se ilustra en las siguientes viñetas:

"Voy a apoyarla hasta donde ella quiera, si quiere estudiar hasta la universidad o sólo la primaria pues la voy a apoyar" (Mamá de niña con trastorno de lenguaje).

"Es un niño muy inteligente, sí puede salir adelante pero necesita mucho trabajo" (Mamá de niño con trastorno generalizado del desarrollo).

"Sé que si le echamos ganas ahorita puede ser un niño normal, lo indispensable son los 12 años de su vida..." (Mamá de niño con hipoacusia).

"La meta es que sea una niña como cualquier otra niña, no quiero que sea la mejor simplemente que sea una niña como cualquier otra y que sea lo que ella quiere ser cuando sea grande, que tome sus propias decisiones" (Mamá de niña con trastorno generalizado del desarrollo).

"De manera alentadora, pues la veo realizada profesionalmente porque ella es muy inteligente" (Mamá de niña con autismo).
El 43\% restante no lo vislumbra positivamente y refieren expresiones como:

"No muy bien definido, aun tengo la esperanza de que algún día pueda hacer sus obras, que pueda estar entretenido en algún tallercito de pintura con sus primos, estar apoyándolo, rodeado de amigos y familiares que también entiendan la problemática" (Mamá de niño con distrofia muscular de Duchenne).

"Yo quisiera que realmente saliera adelante y avanzara, pues pienso que si me pasa algo nadie va a ver por él, nadie me lo va a cuidar" (Mamá de niño con retraso mental).

"Si vivo, pues bien, pero si no, mis hijos me dicen que no me preocupe, que ellos se hacen cargo de él" (Mamá de niño con autismo).

"Quisiera imaginarlo bien pero creo que esto lo va a marcar para todo, porque si yo quisiera tener otro bebe tendría que ser también con esto" (Mamá de niño con epilepsia).

"Lo percibo con mucha necesidad de sus hermanos porque mamá no le va a durar toda la vida, lo percibo con la necesidad de los demás" (Mamá de niño con secuela de encefalopatía hipóxica, neumopatía crónica del lactante, hispoxicoisquémica y gastrotomia).

"Tratar de darle cariño, no hacerla menos. Tratarla igual que a su hermana, no por su problema o estado físico la vamos a hacer menos..." (Mamá de niña con parálisis cerebral infantil).

"Está muy difícil, siento que no va a llegar a algo, a desarrollarse completamente" (Mamá de niño con hemiparesia).

El $81 \%$ de las madres señala aceptar la discapacidad argumentando expresiones como: Así lo quiere Dios, que está acostumbrada a la discapacidad o que es un bien para su hijo. Algunas de estas expresiones se muestran a continuación:

"Se lo dejo a Dios porque yo no puedo adivinar" (Mamá de niño con parálisis cerebral infantil).

"No me lo esperaba pero te vas haciendo a la idea de que quién más para sacarla adelante más que yo, ahora siento que es normal" (Mamá de niña con parálisis cerebral infantil). 
"Ya me acostumbré, que si no es una cosa, es otra, y se debe hacer lo que se debe" (Mamá de niño con hemiparesia).

Dinámica familiar. Respecto al apoyo de la pareja se observa que el $61.9 \%$ lo percibe bueno, el $21.4 \%$ reporta que regular y el $9.5 \%$ lo describe como malo. El 7.2\% no contestó. La teoría plantea la importancia de que la madre se sienta apoyada por su pareja (Bruns \& Foerster, 2011; Sánchez, 2006). Actualmente se proyecta que las investigaciones se diseñen mediante la observación de los cambios en las políticas, iniciativas y prácticas que permitan a las familias manejar el cuidado de sus hijos con discapacidad de manera positiva, respetuosa y con empoderamiento. En este aspecto, la psicología tiene un papel muy importante al fomentar el bienestar psicológico, ayudar a disminuir el estrés de las madres a través de programas para aprender a manejarlo, contribuir al ajuste de la familia ante la discapacidad, brindar asistencia en la solución de problemas, e influir en un adecuado apoyo a las familias tomando en cuenta las necesidades de ésta. Así mismo, se plantea que si se empodera a las madres, éstas pueden lograr salir adelante, a pesar de no contar con el apoyo de la pareja en aquellos casos en que como se observó, no se contaba con ella (Levine, 2009). En este sentido se indagó si las madres percibían que su pareja les ayudaba a sobrellevar la discapacidad del menor; el 71.4\% respondió afirmativamente, mientras que el $23.8 \%$ afirmó que no lo percibía.

En cuanto al apoyo que la madre describe por parte de la familia extensa, la mitad de los casos señala que no es necesario; el 35.7\% sí siente apoyo de ambas familias, y en el $14.3 \%$ ningún integrante de la familia extensa las ha apoyado. En el $88.1 \%$ de los casos las madres consideran que la familia no posee los conocimientos respecto a la discapacidad de sus hijos, lo cual es predecible, ya que los mismos padres no han logrado obtener los conocimientos sobre la discapacidad de sus hijos.

Dinámica comunitaria. Se indagó si las madres consideraban útil el contacto con padres que tuvieran un hijo con discapacidad; el 92.9\% refiere que sí, ya que al estar en contacto con ellos podían compartir experiencias y opiniones sobre la atención del menor, así como aprender de situaciones que los otros ya hubieran vivido y ellas apenas estuvieran enfrentando.

El $61.9 \%$ de las familias considera que el niño es bien percibido por los vecinos; el $26.2 \%$ refiere que no comparte con vecinos, y sólo el $9.5 \%$ considera que es mal percibido. En el $90.5 \%$ no existen problemas en lo referente al niño con los vecinos. Y en cuanto a la actitud que toman los vecinos hacia el niño, en el $57.1 \%$ es normal o buena, el $31 \%$ no comparte con éstos, y el $7.1 \%$ reporta que es mala.

\section{DISCUSIÓN}

Se esperaría que las madres tuvieran un mayor conocimiento e información de la discapacidad de su hijo al estar integradas a un centro especializado en la rehabilitación del menor con discapacidad donde la población no es tan numerosa como en otros sectores de salud en México; sin embargo, los resultados indican que aún no han recibido información.

Se ha observado en estudios realizados por Marcheti et al. (2008); Masood et al. (2007); Pérez y Lorenzo (2007) que si las madres reciben una información adecuada que les brinde los conocimientos sobre la discapacidad de su hijo, se beneficia notablemente a la madre y a la familia en general para que puedan ir elaborando su duelo, modifiquen la imagen errónea del diagnóstico que podría alimentar falsas ilusiones en las madres acerca de una mejoría de la discapacidad, además de que la familia, al tener los conocimientos, puede comenzar a ocuparse del tratamiento del menor. Estos estudios indican que las madres, en general, presentan frustración, tristeza, desdicha o culpa al saber que su hijo tiene una discapacidad, pues al conocer el diagnóstico se rompen los ideales que tienen ellas respecto a su hijo. Se puede observar que los resultados del presente estudio concuerdan con lo encontrado por estos autores; se encuentra también que dichas emociones no se modifican significativamente con el paso de los años, sino que, por el contrario, comienzan a aparecer otras, como la preocupación por el futuro del menor en caso de que ellas como madres lleguen a faltar, lo que a su vez se relaciona con la ausencia de alternativas educativas y sociales viables para ellas y sus hijos.

Por lo anterior, se considera fundamental trabajar con grupos de madres de hijos con discapacidad para que éstas obtengan mayores conocimientos, se les permita expresar emociones positivas o negativas, y se les brinde la contención adecuada para poder favorecer la adaptación del menor y de la familia ante la discapacidad.

Se percibe que más de la mitad de las madres describen que el apoyo de sus parejas en relación con la discapacidad del menor es bueno y casi la mayoría reporta que su pareja le ayuda a sobrellevar la discapacidad; sin embargo, difieren las respuestas cuando las madres reportan, a lo largo de la entrevista, que su pareja pasa poco tiempo con la familia por cuestiones laborales; al ser militares no tienen horarios específicos y muchas veces pasan más tiempo en el trabajo que con ellas y sus hijos. Podemos observar que, a pesar de que se trata de padres ausentes la mayor parte del tiempo, las madres se sienten apoyadas por ellos, conducta que se observa en la población mexicana. 
Más de la mitad de las madres refiere que no ha necesitado ayuda de la familia extensa, lo cual probablemente se deba a la constante movilidad de residencia que presentan las familias de militares. En la mayoría de los casos la familia se encontraba en el Distrito Federal, lugar que no era su estado de origen; esto significa que la familia extensa se encontraba en algún estado del país alejado de sus parientes.

En la dinámica comunitaria se puede observar que casi la totalidad de las madres considera útil el contacto con otros padres, para así poder compartir experiencias, opiniones o aprender de ellos. A pesar de que las madres consideran útil este contacto, éstas no tienen relación con otras familias similares a las de ellas; así mismo, en el CRI no se promueve la relación entre las madres o las familias, lo cual sería importante para poder crear redes de apoyo para éstas. La percepción de las madres referente a cómo es visto el menor por sus vecinos es de que no existen problemas en este sentido, ya que los vecinos ven bien al niño y la actitud que muestran los vecinos es buena $\mathrm{y} / \mathrm{o}$ normal hacia su hijo. La literatura plantea la importancia que juegan estas redes de apoyo en las familias, pues expresan un alto grado de satisfacción ante el apoyo social, difiriendo del apoyo institucional, ya que las familias no se sienten suficientemente apoyadas por esta estructura, lo cual provoca ansiedad e ira en ellas (Bruns \& Foerster, 2011; Steel et al., 2011; Tsibidaki \& Tsamparli, 2007).

Este estudio es un acercamiento para observar el nivel de conocimiento que poseen las madres con respecto a la discapacidad de sus hijos, así como la dinámica familiar y comunitaria que existe. Los resultados tienen varias implicaciones prácticas como la comprensión del problema, un mayor conocimiento de las madres sobre la discapacidad de sus hijos, y especialmente la posibilidad de instrumentar un programa de intervención para las madres de hijos con discapacidad.

\section{REFERENCIAS}

Acle, G., Roque, M., Zacatelco, F., Lozada, R. y Martínez, L. (2007) Discapacidad y rezago escolar: Riesgos actuales. Acta Colombiana de Psicología, 10(2), 19-30.

Anderson, M., Elliott, E. \& Zurynski, Y. (2013). Australian families living with rare disease: Experiences of diagnosis, health services use and needs for psychosocial support. Orphanet Journal of Rare Diseases, 8(22).

Berástegui, A. y Gómez-Bengoechea, B. (2006). Los menores con discapacidad como víctimas de maltrato infantil: una revisión. Intervención Psicosocial, 15(3), 293-306.

Bruns, D. \& Foerster, K. (2011). We've been through it all together: supports for parents with children with rare trisomy conditions. Journal of Intellectual Disability Research, 55(4), 361-369.
Burrows, K., Adams, C. \& Spiers, J. (2008). Sentinels of Safety: service dogs ensure safety and enhance freedom and well-being for families with autistic children. Qualitative Health Research, 18(12), 1642-1649.

Cagran, B., Schmidt, M. \& Brown, I. (2011). Assessment of the quality of life in families with children who have intellectual and developmental disabilities in Slovenia. Journal of Intellectual Disability Research, 55(12), 1164-1175.

Castro, P., Fernández, G., Gómez, A., Campos, I., García, I., Bert, J. y Rodríguez, B. (2008). El maestro y la familia del niño con discapacidad. Cuba: Pueblo y Educación.

Ching, B. (2009). Quality of life of family caregivers of children with autism. Autism, 13(1), 81-91.

Covarrubias, O. (2008). Factores que influyen dentro de la familia, en la aceptación o rechazo de la persona con discapacidad intelectual. Tesis de Licenciatura. UNAM, Facultad de Psicología, México.

De Andrés, C. y Guinea, C. (2012). La atención a la familia en atención temprana: Retos actuales. Psicología Educativa, 18(2), 123-133.

Gargiulo, R. (2006). Special education in contemporary society. An introduction to exceptionality. Estados Unidos: Thomson/Wadsworth.

Gobierno del Distrito Federal, DIF -DF, UNICEF. (2006). El reto de la inclusión y atención integral de niños, niñas y jóvenes con discapacidad en el Distrito Federal. México.

Hernández, R., Fernández, C. y Baptista, P. (2010). Metodología de la investigación. $5^{\text {a }}$ ed. México: Mc Graw-Hill.

Huang, Y., Tsai, S. \& Kellet, U. (2011). Fathers of children with disabilities: Encounters with health professionals in a Chinese context. Journal of Clinical Nursing, 21, 198-206.

Instituto Nacional de Estadística y Geografía (INEGI). (2010). Principales resultados del censo de población y vivienda 2010. Web: www.inegi.gob.mx

Levine, K. (2009). Against all odds: Resilience in single mothers of children with disabilities. Social work in health care. 48, 402-419.

Madrigal, A. (2007). Familias ante la parálisis cerebral. Intervención Psicosocial, 16(1), 55-68.

Marcheti, M., Noda, M. y Ferreira, M. (2008). Experiences of mothers of disabled children: a phenomenological study. Acta Paulista de Enfermería, 21(1), 46-52.

Martínez, M. y Bilbao, M. (2008). Acercamiento a la realidad de las familias de personas con autismo. Intervención Psicosocial, 17(2), 215-230.

Masood, A.; Turner, L. \& Baxter, A. (2007). Causal attributions and parental attitudes toward children with disabilities in the United States and Pakistan. Council for Exceptional Children 73(4) 475-487.

Masten, A. \& Powell, J. (2007). A resilience framework for research, policy and practice. En: Luthar, S. (Ed.) Resilience and vulnerability. Adaptation in the context of childhood adversities (pp. 1-25). USA: University Cambridge Press. 
Maul, C. \& Singer, G. (2009). Just good different things: specific accommodations families make to positively adapt to their children with developmental disabilities. Topics in Early Childhood Special Education, 29(3), 155-170.

Mora, A., Córdoba, L., Bedoya, A. y Verdugo, M. (2007). Características de la calidad de vida en familias con un adulto con discapacidad intelectual en la ciudad de Cali, Colombia. Diversitas, 3(1), 37-54.

Núñez, B. (2008). Familia y discapacidad. De la vida cotidiana a la teoría. Argentina: Lugar Editorial.

Ortega, P., Salguero, A. y Garrido, A. (2007). Discapacidad: paternidad y cambios familiares. Avances en Psicología Latinoamericana, 25(1), 118-125.

Peralta, L. y Arellano, T., (2010). Familia y discapacidad. Una perspectiva teórico-aplicada del Enfoque Centrado en la Familia para promover la autodeterminación. Electronic Journal of Research in Educational Psychology. 8(3), 13391362.

Pérez, V. y Lorenzo, Z. (2007). El impacto del déficit mental en el ámbito familiar. Revista Cubana de Medicina General Integral, 23(3), 1-8.

Programa Nacional para el Desarrollo de las Personas con Discapacidad 2009 - 2012. (2009). Por un México incluyente: construyendo alianzas para el ejercicio pleno de los derechos de las personas con discapacidad. México: Secretaria de Salud/Consejo Nacional para las Personas con Discapacidad.

Rodríguez, M., Alvarado, A. y Moreno, M (2007). Construcción participativa de un modelo socioecológico de inclusión social para personas en situación de discapacidad. Acta Colombiana de Psicología, 10(2), 181- 189.

Rolander, Y., Montes, B. y Culebro, R. (2008). Armonización legislativa en materia de discapacidad en México: actualidad y retos. México: Instituto Mexicano de Derechos $\mathrm{Hu}-$ manos y Democracia, A.C.

Rosenzweig, J., Brennan, E., Huffstutter, K. \& Bradley, J. (2008). Child care employed parents of children with emotional or behavioral disorders. Journal of Emotional and Behavioral Disorders, 16(2), 78-89.

Sánchez, P. (2006). Discapacidad, familia y logro escolar. Revista Iberoamericana de Educación, 40(2), 1-10.

Sarmiento, M. (1994). Psicoprofilaxis familiar. Colombia: Editorial Universidad Santo Tomás de Aquino.
Soto, R. (2008). Adaptación de la forma abreviada del "Cuestionario de recursos y estrés (QRS-F, 1983), para padres de personas con autismo. Actualidades Investigativas en Educación, 8(1), 1-27.

Soulé, G. (2009). Papás especiales para niños especiales. México: Editorial Diana.

Steel, R., Poppe, L., Vandevelde, S., Van Hove, G. \& Claes, C. (2011). Family quality of life en 25 Belgian families: Quantitative and qualitative exploration of social and professional support domains. Journal of Intellectual Disability Research, 55(12), 1123-1135.

Stoner, J. \& Angell, M. (2006). Parent perspectives on role engagement: An investigation of parents of children with ASD and their self-reported roles with Education professionals. Focus on autism and other developmental disabilities, 21 (3), 177-189.

Strnadová, I. (2006). Stress and resilience in families of children with specific learning disabilities. Revista Complutense de Educación, 17(2), 35-50.

Torres, L. y Maia, E. (2009). Percepción de las madres acerca del contenido de la información del diagnóstico de Síndrome de Down. Revista Chilena de Pediatría, 89(1), 39-47.

Tsibidaki, A. \& Tsamparli, A. (2007). Support networks for the Greek family with preschool or school-age disable children. Electronic Journal of Research in Educational Psychology, 5(2), 283-306.

Ulloa, F. (2010). Dinámicas comunitarias y unos parches. Material de partida para el taller intensivo CASCA CUJCUJ. http://es.scribd.com/doc/35072831/ dinámicas-comunitarias-descripción-trampas-y-más

United Nations Statistics Division (UNSD). (2009). Child disability. The State of the World's Children. United Nations Children's Fund.

Vargas, C., Arauza, C., Folsom, K., Luna, M., Gutierrez, L., Ohliger, P., Shelton, K., Foreman, C., Waffle, D., Reynolds, R. \& Cooper, P. (2012). A community engagement process for families with children with disabilities: Lessons in leadership and policy. Matern Child Health Journal, 16, 21-30.

Zipper, I. \& Simeonsson, R. (2004). Developmental vulnerability in young children with disabilities. En: Fraser, M. (Ed.) Risk and resilience in childhood. An ecological perspective (pp. 161-182) USA: NASW Press. 